\title{
El uso del juego dramático en el aula de español como lengua extranjera
}

\author{
Gabino Boquete Martín \\ Universidad de Alcalá
}

Recibido: 10 de febrero de 2013 / Aceptado: 8 de octubre de 2013

ISSN: $1697-7467$

\begin{abstract}
RESUMEN: El objetivo de este artículo es mostrar las actitudes y creencias de los profesores hacia el uso del juego dramático en el aula de ELE, su conocimiento sobre estas técnicas y percepción sobre la pertinencia de su implantación como un elemento metodológico habitual. Se realiza una investigación cualitativa con profesores de español durante un curso de actualización metodológica llevada a cabo en la Universidad de Alcalá. Con este trabajo se muestra la disposición de los profesores al uso del juego dramático por considerarlo motivador y eficaz; sin embargo, no utilizan estas técnicas habitualmente en el aula debido a que se sienten limitados por la realidad de la práctica educativa. Consideran, asimismo, que el componente lúdico es compatible con el rigor académico y la disciplina en el aula.

Palabras clave: juego dramático, Español como Lengua Extranjera, componente lúdico, componente comunicativo.
\end{abstract}

\section{The use of Drama Techniques in Spanish as a Foreign Language Classroom}

ABSTRACT: The aim of this article is to show the attitudes and beliefs of teachers toward the use of dramatic play in the language classroom, their knowledge of these techniques and perception of the relevance of its implementation as a methodological element. For this, a qualitative research is performed with Spanish teachers attending a refresher course conducted at the University of Alcalá. This work shows the willingness of teachers to use dramatic techniques and how they consider them effective and motivating; however, teachers usually do not use these techniques in the classroom because they feel constrained by the reality of educational practice. They also consider that the playful component supports academic rigor and discipline in the classroom.

Keywords: drama techniques, Spanish as a Foreign Language, playful component, communicative component.

\section{INTRODUCCIÓN}

La incorporación de métodos comunicativos a la enseñanza de lenguas ha ayudado de modo importante a que se utilicen técnicas dramáticas, tales como juegos de rol, mímica, improvisaciones, simulaciones, etc. Por ello se plantea la posibilidad de encontrar en las actividades teatrales una metodología que ayude a los alumnos a comunicarse con fluidez y corrección; estas actividades pueden ser útiles, así mismo, para interpretar y comprender críticamente todo tipo de textos y para conocer los aspectos fundamentales del medio sociocultural propio de la lengua estudiada. 
Es oportuno aclarar que la aplicación de estas técnicas teatrales no tiene como fin último la consecución de talleres de teatro o de actuación (lo cual es, de hecho, una experiencia muy gratificante y de probada eficacia en la enseñanza de lengua y literatura): la misión es la de formar a los estudiantes de lengua española en la práctica comunicativa mediante la incorporación de estrategias, inspiradas en el juego dramático, que sean el eje que articule el programa y defina el estilo de las actividades. Se trata de conseguir una mejora en la competencia comunicativa de los estudiantes y crear un espacio donde llevar a cabo situaciones cercanas a la realidad (en las que recrear aspectos culturales y sociales del ámbito hispano que, de otro modo, sería muy difícil de conseguir) y que permitan al estudiante poder equivocarse sin miedo a hacer el ridículo.

El uso del juego dramático en el aula permitirá a los alumnos mejorar, de manera sensible, su pronunciación en español, sus habilidades comunicativas y su conocimiento de la realidad social y cultural españolas. Este artículo quiere mostrar la pertinencia de la integración del juego dramático en la enseñanza de lengua; se pretende estudiar cómo su aplicación afecta al proceso de aprendizaje, su impacto sobre la mejora de la competencia comunicativa y los elementos positivos que aporta a la motivación, su repercusión en las estrategias de enseñanza y aprendizaje, así como la interrelación entre los participantes en el aula.

Para ello se realizará, dentro del marco teórico, una descripción de los conceptos "drama" y "teatro", las características del juego dramático y sus posibilidades de utilización en el aula de lengua. Se llevará a cabo, además, un estudio cualitativo con profesores de español, en el que, mediante entrevistas y la realización de ejercicios prácticos, así como el reporte de su desarrollo, se analizará sus actitudes y creencias sobre el uso del juego dramático en el aula de ELE.

El desarrollo de la investigación tiene un carácter esencialmente práctico y parte de la idea de que la clase es un "drama", en el sentido de "hacer y realizar", acción en esencia: todos los ejercicios propuestos son usados habitualmente por este autor en su docencia, tanto en la enseñanza a estudiantes de español como a profesores de ELE; son parte activa y resultado de una tesis doctoral, Boquete (2012), desarrollada según la metodología de "investigación-acción en el aula" y que está centrada en el uso del juego dramático como elemento integrador en la enseñanza de lenguas. La reflexión final de esta tesis doctoral aporta las pautas adecuadas para la programación de un curso monográfico ${ }^{2}$, en el que el uso de técnicas dramáticas tales como juegos de rol, mímica, improvisaciones, simulaciones, etc., son parte esencial en el diseño de las unidades didácticas.

\section{El uso del Juego dramático en el aula}

La utilización de juegos para la enseñanza/aprendizaje de lenguas extranjeras es ya un recurso antiguo que se ha ido consolidando y extendiendo con el tiempo. Los juegos constituyen una forma amena de practicar algo tan arduo como el aprendizaje de una lengua

${ }^{1}$ Los postulados establecidos en la "investigación-acción en el aula" desarrollados por Elliott y Stenhouse (entre otros investigadores) están dirigidos a solucionar problemas diagnosticados en situaciones específicas, para mejorar la realidad educativa, mediante la reflexión y la colaboración de los participantes (los propios estudiantes y profesores observadores, con los que se intercambian experiencias).

${ }^{2}$ Curso monográfico sobre la prosodia en español para estudiantes de cualquier nacionalidad y con un nivel B2. Ver: Boquete (2012: anexo I, Propuesta final). 
extranjera y añaden la variedad necesaria para mantener el interés y el esfuerzo en esta tarea. Fernández (1997) afirma que el juego es una forma innata de aprender; es la capacidad que tiene el ser humano de experimentar y apropiarse de lo que le rodea de una forma placentera. El aprendizaje verdadero es siempre un juego en el que entran la motivación, el deseo, el reto, el descubrimiento, la creatividad, el placer de llegar al final y haberse superado, etc. Es un mecanismo de adaptación y aprendizaje que integra vías de conexión con la cultura y la realidad en la que se está inmerso.

El juego como elemento de aprendizaje permite caer en el error sin consecuencias negativas, por lo que ayuda al desarrollo personal de cada individuo, le permite realizar el ensayo sin riesgos y le pone cada vez tareas más difíciles, problemas que no sabe solucionar $\mathrm{y}$ que acomete con el juego porque sabe que puede combinar y probar sin compromiso.

Centrándonos en el juego dramático, ya desde la infancia, el ser humano recurre este elemento lúdico para descubrir el mundo y comunicarse con los demás, como parte del proceso de socialización, gracias a la imitación de los adultos, sus acciones, sus gestos y su lenguaje. Antes de saber hablar, el niño intenta interrelacionarse con los demás, comunicar sus necesidades y comprender los mensajes que se le dirigen, a pesar de su desconocimiento de los mecanismos del lenguaje y la estructura del sistema. Primero aprende los usos y las funciones de la comunicación y mucho más tarde el código en sí mismo; la primera forma de comunicarse surge de la imitación, y su repertorio inicial está relacionado con las funciones del lenguaje que tienen que ver con las acciones cotidianas: alimentarse, expresar estados de ánimo, reclamar la atención de los demás, etc. Por ello, el juego se establece como una actividad de enorme importancia tanto en la adquisición del lenguaje como en la ampliación de la competencia lingüística (Robles, 2007).

Puesto que, desde los primeros meses de vida, el ser humano elabora juegos en los que recrea situaciones, conductas y papeles sociales que le ayudan a descubrir la realidad que le rodea y a utilizar su lengua materna, el uso del juego dramático podrá ser útil en el aprendizaje de una lengua extranjera. Estas actividades serán oportunas para adquirir los conocimientos que faciliten la comunicación en la lengua meta y ayudarán al estudiante a tener seguridad y confianza en sí mismo.

\subsection{Drama y teatro}

A menudo se tiende a confundir los conceptos drama y teatro. Magariño (1996: 153) hace constar que:

"Drama y teatro" son términos que se han empezado a introducir en la educación como forma efectiva de enseñanza, como ayuda para profundizar y descubrir el mundo que nos rodea, y como medio de ahondar en la personalidad del individuo.

Es cierto que ambos términos pueden ser considerados sinónimos relacionados con obras de teatro, actores, ensayos y representaciones en público. Aunque, siguiendo con Magariño (1996: 154), el teatro está más estrechamente vinculado con la idea de representación o puesta en escena ante un público, mientras que drama tiene más que ver con "hacer y realizar" y plantea, por lo tanto, una relación más directa con el mundo de la didáctica y la enseñanza de lenguas enfocado desde la perspectiva del estilo comunicativo. 
En las actividades dramáticas el alumno aporta su propia personalidad y experiencia en el proceso de aprendizaje, utilizando la capacidad innata en el ser humano para imitar, hacer gestos, proyectarse en otros personajes y expresar ideas a través de su cuerpo y de su voz. La idea de drama se asocia a la acción que se desarrolla en el momento, por primera vez, y de manera irrepetible, mientras que el teatro estaría más relacionado con la representación de una obra escrita.

Mediante el drama, el alumno puede experimentar con la lengua meta en actividades donde se desarrolle la habilidad de enfrentarse a situaciones reales de un modo diferente al de la memorización de diálogos procedentes de un libro. Esto no es óbice para aceptar la validez de la representación de una pieza teatral como recurso pedagógico con el que se puede fomentar la lectura expresiva, por ejemplo. La lectura enseña a realizar las pausas en los lugares oportunos y adaptar la voz a las entonaciones adecuadas para lograr transmitir las ideas, el sentido y el sentimiento expresado en el texto. Se requiere una buena lectura para dar vida a unos textos que no se deben considerar un modelo conversacional puro (Fons, 2010). Por ello, y a pesar de asumir la opción de realizar puestas en escena teatrales, se concebirá la conversación como algo mucho más libre e imprevisible en una situación real que la de una obra teatral.

En este artículo el concepto juego dramático hará referencia al significado original griego de drama (hacer), más que al arte de representar una acción determinada en un escenario. El juego dramático tiene una dimensión lúdica, mientras que del teatro se toman las técnicas que sirven para poner en práctica el drama como acción dialogada entre los personajes; se entiende, así mismo, como un proceso de recreación de situaciones con el deseo de conocer $\mathrm{y}$ aprender, en este caso concreto, una lengua.

\subsection{Juego dramático vs. espectáculo teatral}

Eines y Mantovani (1997) exponen como diferencia fundamental entre los dos campos el hecho de que el de teatro plantee una representación en la que interesa el resultado final o espectáculo, mientras que el concepto que se propone como punto de partida de este estudio, juego dramático, plantea un proyecto de desarrollo de la capacidad comunicativa del estudiante de lengua, centrándose en el proceso o la realización del proyecto que ha propuesto el grupo o clase.

En la actividad teatral convencional las acciones y los personajes son dirigidos por el profesor/director, mientras que el estudiante/actor memoriza el texto y acepta la propuesta sin plantear opciones. El papel del director/profesor se centra en la motivación general del grupo, en el que los actores/estudiantes son guiados por el profesor en situación de juego colectivo. Los actores y espectadores son intercambiables y los papeles (roles) son elegidos por los jugadores. El profesor hace que la acción avance y se desarrolle; con sus intervenciones encauzará los problemas que vayan surgiendo y las dificultades de la acción.

En el juego dramático el ejercicio puede darse por concluido en cualquier momento, sin que se un establezca un límite previo; del mismo modo, es posible finalizar la actividad si no se consigue un desarrollo de la actividad adecuado a la propuesta planteada. Además, se puede poner en práctica en cualquier espacio mientras que un espectáculo teatral está condicionado por los escenarios que se quieran poner en escena. Esto es fundamental en el desarrollo real de las clases de lengua: profesores y estudiantes van a tener que trabajar, generalmente, en un espacio limitado y condicionado por el mobiliario del aula. 


\subsection{Características del juego dramático}

Una vez establecidas las diferencias entre espectáculo y juego dramático se van a enumerar sus características y las razones que apoyan su uso en la clase de enseñanza de lenguas.

\subsubsection{Genera actitudes positivas hacia la materia y aumenta la motivación}

La utilización del juego dramático en la enseñanza de lenguas busca la motivación del estudiante y la del profesor, de modo que favorece el desarrollo de la confianza y de la autoestima del alumno (y, por qué no, la del profesor), ya que se trata de una experiencia satisfactoria que proporciona un alto nivel de implicación emocional e intelectual mediante la participación activa; fomenta la confianza del alumno en sí mismo y en su capacidad para poder comunicarse, ya que se valora la comunicación efectiva por encima de la comunicación correcta; es una forma eficaz de amortiguar el sentimiento de error y la frustración que ello conlleva; desarrolla la fantasía y anima a los estudiantes a usar la lengua de una forma creativa; ofrece al alumno la posibilidad de utilizar la lengua libremente con actividades abiertas donde los alumnos no se limitan a responder o rellenar, sino que deben poner en juego la decisión y la toma de iniciativa (Cómitre y Valverde, 1996).

El uso del juego dramático favorece la creación de actitudes positivas hacia la asignatura en que se emplea, pero supone, además, una mejora hacia las clases en general, hacia el centro escolar y hacia el grupo al que pertenecen los sujetos y sirven como elemento de disfrute en el aula. Para Motos (1993: 75):

Ello significa que los sujetos disfrutan con las actividades que realizan y encuentran placer en ellas. Por lo tanto, los procedimientos didácticos basados en el lenguaje dramático estimulan a que los sujetos disfruten con las actividades que están realizando.

\subsubsection{Ayuda a crear una dinámica eficaz de grupo}

El juego dramático colabora a la formación de grupos compactos, en un clima de cooperación, gracias a dinámicas y hábitos de grupo. Las actividades teatrales fomentan la destrucción de las barreras individuales y permiten conocer al otro en mayor profundidad. El alumno interrelaciona con sus compañeros y coopera con ellos para comunicarse en el contexto adecuado, aprende a relacionarse con ellos y desarrolla estrategias para ello, dentro del cumplimiento de una serie de reglas establecidas.

Desde el punto de vista del individuo, estas actividades permiten atender al alumno en particular, teniendo en cuenta sus intereses, aficiones y experiencias. A su vez, cada alumno aporta algo de sí al grupo con la expresión de su personalidad, sus reacciones y su relación con los demás, lo que supone un enriquecimiento personal y la creación de un clima de trabajo en equipo, a partir del cual es más fácil y motivador el aprendizaje de una lengua (Cómitre y Valverde, 1996). 


\subsubsection{Herramienta útil para la comunicación}

Las actividades de dramatización crean situaciones que permiten a los aprendices interaccionar de una manera natural; gracias a la negociación del significado que suele darse en las improvisaciones, los estudiantes investigan sobre los conceptos que posee de la lengua meta y afianzan los que ya se tienen de ella.

\subsubsection{Reflejo de la realidad social y cultural del momento}

El juego dramático puede servir para recrear situaciones de la realidad exterior del aula, lo cual ayuda a atenuar la artificiosidad de muchos de los materiales didácticos y de las actividades lingüísticas utilizados en el aula. El teatro facilita el encuentro de los estudiantes con una variedad de experiencias lingüísticas, situadas en contexto, que difícilmente podrían estar disponibles de otra manera (Cómitre y Valverde, 1996).

Cuando juegan a imitar situaciones, los alumnos experimentan con la aplicación de diferentes registros sociales y toman conciencia del estrecho vínculo que existe entre lengua y contexto. Es idóneo para producir un lenguaje auténtico y hacer un uso real del mismo; ayuda al alumno, asimismo, a comprobar la eficacia de las estructuras gramaticales y el léxico que se estudian en estas sesiones.

\subsubsection{Medio de apoyo en los enfoques por tareas}

El juego dramático complementa la metodología del enfoque por tareas. Como preparación para la tarea final (la representación teatral), el alumno realiza una serie de tareas intermedias o facilitadoras (juegos de exploración dramática, improvisación, mímica y voz, análisis de la obra, orientaciones en la puesta en escena, etcétera).

\subsubsection{Colabora en la mejora de la expresión oral}

Ayuda a desarrollar la fluidez en el trabajo de las destrezas lingüísticas, sobre todo en la expresión y comprensión orales, algo primordial en el aprendizaje de una lengua y que la enseñanza tradicional desatiende a menudo. Además, la variedad y flexibilidad de las actividades basadas en el juego dramático pueden ser de gran utilidad a la hora de plantear el estudio de los contenidos teóricos. La aplicación de técnicas teatrales ofrecen un completo repertorio de ejercicios y actividades de articulación, respiración, proyección y entonación que pueden emplearse para, de acuerdo con Hayes (1984: 41):

Incrementar la conciencia de los estudiantes sobre su propia voz y ayudarles a ser más flexibles en su uso del habla.

Estas técnicas pueden ser una herramienta útil para el estudio de aspectos suprasegmentales de la pronunciación (como la entonación, el acento, el ritmo, la calidad de la voz, etc.). La entonación, por ejemplo, es fundamental para comprender la intención del hablante, tal y como manifiesta Brown (1994: 240): 
(...) no solo para la interpretación de enunciados declarativos e interrogativos, o cuestiones de énfasis, sino también para la interpretación de mensajes más sutiles como el sarcasmo, el cariño, el insulto, el elogio...

En el proceso de desarrollo del juego dramático, y como parte de la construcción del personaje, los alumnos deben adaptar el papel a su personalidad, trabajando con distintos patrones de entonación, sonidos y vocalizaciones. Estos ejercicios pueden servir de motivación para que el estudiante mejore su pronunciación, a la vez que investigan la incorporación de los elementos no verbales en el proceso de comunicación.

Hayes (1984: 100), asegura que:

Una ventaja de que los estudiantes aprendan sus papeles es que esto les da una oportunidad para concentrarse y trabajar sobre la voz y la pronunciación de una forma más interesante que a través de drills o repeticiones mecánicas en clase. Cada enunciado puede examinarse desde el punto de vista de la articulación, acento, entonación, etc. y la adecuación del estilo de habla de acuerdo con la situación y el rol desempeñado.

Motos (1993: 85-89) comparte esta idea y afirma que los ejercicios inspirados en técnicas dramáticas:

(...) influyen en la expresión oral de los sujetos desarrollando la fluidez verbal; estimulan la adquisición de destrezas para la exposición de los contenidos de una manera secuencializada; y favorecen un mayor grado de imaginación en las producciones.

\subsubsection{Ayuda al desarrollo del conocimiento intercultural}

Continuando con Hayes (1984: 11), a través del teatro:

Podemos explorar cuestiones sociales importantes y, quizá volver a evaluar nuestras propias posturas frente a ellas mientras empezamos a comprender a las otras personas.

\subsubsection{Desarrolla los mecanismos cognitivos}

Este tipo de actividades ejercitan la memoria, la observación y la concentración, habilidades básicas en el aprendizaje de una lengua, y favorecen el uso de la lengua con un fin concreto, tal y como ocurre en el habla real, para poder comunicarse de una forma eficaz y lograr un objetivo preciso (Motos y Tejedo, 1987).

\subsubsection{Refuerza el carácter multidisciplinar del aula}

Gracias a la incorporación de textos literarios se puede ofrecer conocimiento no solo relacionado con elementos lingüísticos, sino que pude ser un complemento ideal para la formación en asignaturas como literatura, historia, etc. (Cómitre y Valverde, 1996). 


\subsubsection{Refuerza la relación personal entre profesores y estudiantes}

La realización de actividades dramáticas hace que el pensamiento de los alumnos sobre el profesor cambie en los aspectos relativos a la función, actitud y estilo de enseñanza del profesor (Motos, 1993: 75).

En cuanto a los aspectos socio-afectivos que se favorecen y estimulan durante las clases de Lengua mediante el uso de procedimientos dramáticos, el autor enumera los siguientes:

(...) la superación de la timidez y la pérdida de la vergüenza para hablar y actuar en público, disminuyen el sentimiento de ridículo, liberan de las clases normales, y provocan relajación. Y por otra parte, las actividades dramáticas proporcionan la oportunidad de realizar actividades y tareas pertenecientes a los diferentes planos de la persona, psicomotor, cognitivo, afectivo y social.

\subsection{Capacidades desarrolladas}

El uso del juego dramático en la enseñanza de lenguas extranjeras tiene como misión el desarrollo de las capacidades intelectuales, lingüísticas, afectivas, sociales, etc., que todo estudiante ha de tener para alcanzar una comunicación plena con los demás hablantes.

Estas capacidades, cuyo objetivo principal es atender a la función comunicativa del lenguaje, de acuerdo con el trabajo realizado por Arroyo (2003:60), pueden resumirse en las siguientes: capacidades cognitivas, conocimiento de uno mismo y de sus propias posibilidades y ejercitación en el aprendizaje; capacidades afectivas, desarrollo de la autoestima y de la expresión libre de los sentimientos en un clima de cordialidad y de trabajo en grupo; capacidades lingüísticas relacionadas con la comunicación y la expresión del lenguaje oral; desarrollo del vocabulario y desarrollo de la lógica en la construcción de frases; capacidades sociales, de comunicación y representación.

\subsection{Estrategias de aprendizaje relacionadas}

El uso del juego dramático pone en funcionamiento el uso de diferentes estrategias; las más importantes podrían ser, según Robles (2007:16-17):

- Planificar lo que se va a decir y los medios para decirlo; ensayar e intentar nuevas combinaciones y expresiones.

- Aprender a sustituir una palabra por un término equivalente, utilizar circunloquios y paráfrasis para suplir carencias de vocabulario y definir algo concreto cuando no se recuerde la palabra exacta.

- Escuchar atentamente para intentar captar las ideas principales; identificar por el contexto palabras desconocidas, identificación de vacío de información y de opinión y valoración de lo que puede darse por supuesto.

- Planificar los intercambios y los turnos de palabra.

- Pedir ayuda a un compañero la hora de formular algo.

- Técnicas de memorización (asociación, contextualización, etc.).

- Uso de gestos para solucionar problemas de comunicación. 


\section{Metodología de LA investigación}

Como complemento a lo expuesto en el capítulo dedicado al marco teórico, conviene aportar estudios que demuestren la eficacia del juego dramático en el aula. Sirva como ejemplo el trabajo de investigación de Motos (1993: 75), que en su artículo sobre la aplicación de las técnicas dramáticas en el aula de Lengua y Literatura manifiesta:

Los resultados constatan que las técnicas dramáticas (juegos, actividades de sensopercepción, sonorización, dramatización y role playing) hacen cambiar favorablemente el significado del concepto que los sujetos tienen de la asignatura Lengua Española, cargándose de connotaciones positivas en las dimensiones semánticas de seguridad, potencia, clima y evaluación. Y también favorecen la creación de actitudes positivas hacia las clases en general, hacia el centro escolar y hacia el grupo al que pertenecen los sujetos.

Motos expone los resultados de una investigación centrada en demostrar que las técnicas dramáticas, cuando se aplican a la enseñanza de la Lengua y Literatura, provocan en los alumnos un cambio de actitud ante ella. ${ }^{3}$ Mediante la combinación de métodos cuantitativos y cualitativos intenta demostrar sus hipótesis, ayudándose de test, grabaciones, inventarios y entrevistas individualizadas y colectivas. A partir del análisis de los resultados, Motos (1993: 85-89) muestra cómo los participantes en estas actividades destacan los siguientes aspectos positivos:

(...) proporcionan diversión; las actividades y tareas propuestas son variadas, nuevas e interesantes; ayudan a desinhibir y a relajar la tensión que se produce normalmente en las clases; estimulan la imaginación; incrementan la empatía entre los compañeros y ayudan a desarrollar la capacidad de razonamiento y la expresión oral.

Este estudio, a pesar de estar centrado en los estudiantes de lengua materna, es extrapolable a la enseñanza de segundas lenguas y sus conclusiones pueden ser aplicables a una clase de español como lengua extranjera. Asimismo, parece oportuno mencionar los vínculos existentes entre ambas áreas de la lengua (L1 y L2 o lengua extranjera) y de la posibilidad de compartir técnicas, estilos de enseñanza, tipos de actividades y, muchas veces, contenidos.

Desde este punto de partida diseñamos un trabajo de 'investigación cualitativa' centrada en preguntas realizadas a profesores de español con el fin de conocer su percepción sobre el uso del juego dramático en el aula. En cuanto al análisis de las respuestas, se utilizará el 'modelo de Likert'.

\section{1. Ámbito de investigación}

La experiencia tuvo lugar durante un curso de formación de profesores de ELE organizado por el Instituto Cervantes e impartido en Alcalingua Universidad de Alcalá durante el

${ }^{3}$ Para ello el autor parte de la investigación llevada a cabo en un aula de 40 estudiantes de Primero de BUP (actual bachillerato): en 14 clases de Lengua durante los cursos académicos 87-88 y 88-89 del Instituto de Bachillerato del Complejo Educativo de Cheste (Valencia). 
mes de abril de 2012, titulado La interacción y motivación: dinámica de grupo ${ }^{4}$. El grupo estaba formado por 24 profesores, en la gran mayoría españoles. En este curso coinciden participantes de muy diferente origen formativo: la mayoría son filólogos (7 de Hispánicas, 3 de Inglesa y 1 de Italiana), y maestros (4); sin embargo, aparecen licenciados en Sociología (1), Historia (3), Derecho (1), Bellas Artes (1) y Psicología (1) y Publicidad (1). ${ }^{5}$

En cuanto a la experiencia en la enseñanza de lenguas, la muestra es bastante equilibrada, con 10 profesores en ejercicio (entre ellos los 4 maestros) y 4 participantes con experiencia en colaboraciones en ONG; el resto, 10 participantes, está en formación o tienen poca experiencia.

El análisis de los datos recabados en las encuestas, basados en 'Tablas de frecuencias', se realiza utilizando el paquete estadístico $\mathrm{SAS}^{6}$ versión 9.1.

\subsection{Relato y secuencia de los ejercicios prácticos experimentados con los profesores}

La primera parte del taller práctico servirá para analizar la respuesta del profesor ante una propuesta práctica y real sobre el uso del juego dramático en clase de lenguas. Para ello, vamos a poner en práctica el ejercicio Conocer, descubrir, secuencia de ejercicios basada en técnicas teatrales para potenciar la memoria sensitiva y para ayudar en la interacción entre los componentes del grupo; es una propuesta de Bercebal (1998), centrada en la formación de actores, adaptada por nosotros a la enseñanza de ELE para el primer día de clase. Suele ser adecuado para romper el hielo y establecer pautas de trabajo basadas en la confianza y colaboración dentro de un grupo ${ }^{7}$.

La sesión comienza en el pasillo, fuera del aula, donde se ha creado cierta expectativa al tener cerrada el aula y no permitir la entrada a los participantes. Es preferible realizarlo antes de que los participantes hayan visto el espacio y, a ser posible, antes de que hayan tenido el primer contacto entre ellos. Comienza la primera parte de la sesión:

- Visualización del espacio donde se va a entrar (imagen inventada).

- Descripción del espacio, de la atmósfera y de sensaciones.

Los participantes colaboran en este primer momento, no parece que nadie se encuentre a disgusto; solo se sienten un poco sorprendidos por la forma de iniciar la clase, ya que se suponían sentados en su mesa, en espera del comienzo de la sesión.

La actividad se traslada al interior del aula: una vez dentro se invita a los participantes a que recorran el espacio y lo comparen con la imagen inventada; en el recorrido se irán comentando estas diferencias con el resto. A continuación se reparte el grupo en parejas, con el fin de que se muestren el espacio unos a otros y de contrastar las diferencias entre el espacio inventado y el que realmente se han encontrado.

\footnotetext{
${ }^{4}$ La programación y contenidos puede ser consultada en: http://cfp.cervantes.es/actividades_formativas/cursos/cursos_instituciones/P733-13.htm

${ }^{5}$ El ámbito de esta encuesta, de los participantes en el curso de formación de profesores de ELE, nos indica la gran variedad de carreras universitarias que convergen. Una de las razones es la situación laboral actual; este elemento no atañe a nuestro estudio, pero sería una situación interesante para analizar.

${ }^{6}$ Statistical Analysis System, SAS Institute Inc.

${ }^{7}$ El desarrollo completo de la sesión está disponible en Boquete (2012: anexo II pág. 87-98).
} 
Antes de ello se habrá hecho una pequeña pausa para anticipar: ¿cómo será la persona con la que cada uno va a formar pareja? Su aspecto físico, su ropa, su voz... El coordinador ayuda a describir todos estos aspectos y conduce las descripciones hacia aspectos más psicológicos. Es muy importante que el trabajo en parejas o en grupos pequeños no se limite al entorno habitual de los participantes, por lo que el coordinador debe cohesionarlos y crear el hábito de trabajo alternado con cualquier compañero.

Se plantea la actividad de Los lazarillos. En este ejercicio un estudiante guía a otro que tiene los ojos tapados, para que no pueda ver, y le va mostrando los objetos y el espacio del aula con el fin de realizar descripciones sensoriales diferentes a las relacionadas con el sentido de la vista. Por parejas, uno hace de ciego, con antifaz, y el otro es su lazarillo. Le irá guiando por el espacio haciéndole observar, con el resto de los sentidos, los elementos del espacio. El profesor, cada cierto tiempo, recuerda a los ciegos y lazarillos un sentido, con el que tienen que trabajar con más intensidad. 'oído, tacto, olfato, gusto'. Se intercambian los papeles para que todos participen desde las dos perspectivas, las del guía y las del ciego.

Pues bien, en general el grupo mostró capacidad de improvisación y de juego, se dejó llevar por las indicaciones y por el ambiente de camaradería necesario para avanzar en la secuencia; asimismo, dejó aflorar el sentido del humor como resultado de las situaciones creadas en el desplazamiento de las distintas parejas por el espacio disponible.

De vuelta a la actividad, se invita a los participantes a que se presenten entre ellos: nombre, edad, procedencia, detalles interesantes de la familia o aficiones, etc. A continuación, se forman parejas mirándose frente a frente. Se pide a los alumnos que traten de ver en su pareja un animal, en el más natural sentido de la palabra. Tras decírselo mutuamente y justificar la elección, se cambia de parejas y se repite. Tras tres o cuatro cambios, se pone en común lo que a cada uno le han dicho y si ha habido similitud entre lo que han opinado distintas parejas o no. Este ejercicio es típico de las clases enseñanza de lenguas; en ese sentido no se está aportando nada nuevo. Lo que, quizá, puede ser novedoso es el intento de incorporarlo a una secuencia más larga en la que los participantes van a tener que desplegar todo su conocimiento descriptivo, tanto de aspectos físicos como psicológicos, y en la que su imaginación va a ser la vía para mecanizar el léxico compartido por el grupo. Además, se añade un elemento dinamizante a un ejercicio que, generalmente, suele realizarse sentado y con la guía de un manual o un diccionario.

El factor tiempo es determinante en esta sesión evaluativa, ya que forma parte de un taller con una extensión limitada a dos horas en la que se deberán estudiar más elementos relacionados con la interacción y la motivación en el aula. Por ello, se da por concluida (a pesar de que este desarrollo puede realizarse durante varias clases y alargarse según las necesidades del programa) y se manda sentar a los profesores.

En un ambiente más convencional pasamos a exponer la opción que queremos presentar como contraste a lo realizado hasta el momento, y que consistirá en mostrar la forma típica de realizar un primer día de clase, centrado en los manuales, en la explicación de las fórmulas de cortesía y la presentación de programas académicos; de la misma manera, se mostrará ejercicios paradigmáticos de descripción que suelen aparecer en los manuales de enseñanza de ELE. Esta secuencia constará de los siguientes pasos: 
Primer día de clase: presentación, formas de cortesía, carteles con el nombre del estudiante encima de la mesa, etc.

Se muestran ejercicios típicos de descripción de varios manuales y niveles: Alonso y Prieto (2011) B1, Álvarez (2000) B2, Castro (2005) A2 y Menéndez (2009) B2; además se reproduce las audiciones relacionadas que los acompañan.

Se presentan ejercicios que aparecen en páginas educativas de Internet, tales como $T o$ doele.net ${ }^{8}$ y se analiza el formato. Se trata de un ejercicio denominado Rompiendo el hielo, en el que se presentan objetivos similares al planteado en la secuencia Conocer y descubrir expuesta anteriormente; las funciones lingüísticas que se plantean en Rompiendo el hielo son similares: dar/pedir información, expresar gustos, saludar y presentarse.

Se completa la información sobre Internet con ejercicios que aparecen en "blogs" educativos, como puede ser el caso de El tinglado ${ }^{9}$. En él se puede encontrar una sección que encaja con las funciones y los objetivos que se están trabajando en el análisis de estilos; se trata de Nortxu, basada en ejercicios supuestamente interactivos en los que practicar con un personaje virtual.

Una vez expuestos y analizados los dos estilos de desarrollo de una secuencia didáctica se procederá a evaluar y expresar preferencias mediante la realización de encuestas.

\section{Actitudes y CReencias de los profesores hacia el uso del Juego DRAMÁTICO EN EL AULA}

Veamos, pues, los resultados que muestra la encuesta, en cuanto al análisis de estilos de enseñanza en la que se plantea la siguiente cuestión:

Pregunta 1: Durante la sesión se han visto dos estilos diferentes de crear un primer encuentro:

A. Un estilo "tradicional" en el que el que cada uno se presenta, cuenta las razones por las que ha decido estudiar español y describe su familia, amigos, etc.

B. Mediante el uso de técnicas teatrales adaptadas a la enseñanza de lenguas, elementos de improvisación y recreación de situaciones, lo que podríamos llamar "juego dramático".

Por favor, elige una de las dos opciones y añade los comentarios que creas necesarios.

\footnotetext{
${ }^{8}$ Disponible en: http://www.todoele.net/actividades/Actividad_maint.asp?s_keyword=\&s_gramatica=\&s_fu nciones $=$ Saludar $+y+$ presentarse $\&$ s cultura $=\&$ Actividad $\mathrm{id}=123$

${ }^{9}$ Disponible en: http://www.tinglado.net/?id=nortxu . En su presentación plantea lo siguiente: “Aquí podrás hablar con mi amiga Nortxu y practicar tu español. Para el aprendizaje de idiomas es esencial que se produzca interacción entre el emisor y el receptor de los mensajes. Una buena manera de ejercitarse es practicar con un personaje virtual, pues están siempre disponibles, eliminan el temor a equivocarnos y pueden simular muy bien contextos reales de comunicación..."
} 


\begin{tabular}{|l|c|c|}
\hline & A & B \\
\hline En general, ¿cuál de los dos métodos te parece más eficaz? & & \\
\hline En general, ¿cuál es el más sencillo de llevar a la práctica? & & \\
\hline ¿Cuál consigue una mayor interacción entre los participantes? & & \\
\hline ¿Cuál consideras que es más motivador para los participantes? & & \\
\hline ¿Cuál preferirías utilizar en clase? & & \\
\hline OBSERVACIONES: & & \\
\hline
\end{tabular}

Los resultados coinciden con las opiniones vertidas durante el debate posterior.

\section{Contraste de estilos de enseñanza}

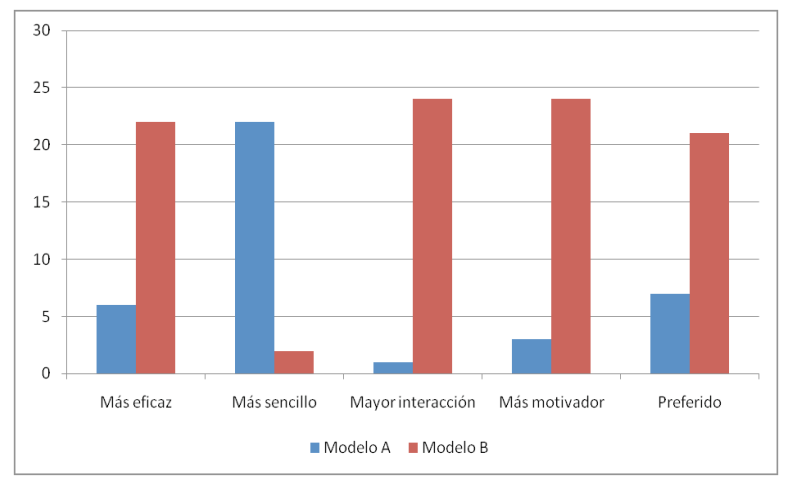

Procedamos a realizar el análisis de la información aportada por los participantes:

- El 72,7\% de los participantes considera que el modelo correspondiente al uso del juego dramático en el aula es más eficaz.

- Sin embargo, el 91,7\% considera el modelo A, el modelo más tradicional, es, a su vez, más sencillo de aplicar.

- El 95,8\% reconoce en el modelo B un modelo más interactivo.

- Para el 87,5\% el modelo B es más motivador.

- Esta distancia entre el modelo A y el B se recorta a la cuando se pregunta por la preferencia, por parte del profesor, a la hora de ser utilizada en clase: el $66,7 \%$ estaría a favor de la práctica del modelo $\mathrm{B}$.

Los comentarios de los profesores intentan puntualizar las afirmaciones:

- Varios profesores abundan en la necesidad de precisar el grupo, la edad, la cultura, etc. Un participante menciona el aula y sus características. 
- Otros coinciden en que ambos estilos son compatibles y que es bueno combinarlos.

- Un profesor teme que el juego dramático pueda ser incómodo para algún estudiante el primer día de clase.

- Se da la situación de profesores que prefieren utilizar el sistema A (más tradicional) en clase a pesar de que considere la técnica B mejor en general.

- Hay, así mismo, mención a la originalidad y a su eficacia a la hora de "romper el hielo y quitar un poco la timidez".

A continuación se pregunta a los participantes quiénes de ellos han puesto en práctica en sus aulas el estilo B. Esta es la situación:

Pregunta 2: ¿Has utilizado alguna vez el estilo B en tus clases/prácticas?

$$
\begin{array}{lll}
\text { NO } & 13 \text { participantes } & 61,9 \% \\
\text { SÍ } & 8 \text { participantes } & 38,1 \%
\end{array}
$$

Dominan, con un $61,9 \%$, los profesores que manifiestan no haber utilizado el estilo B, el uso del juego dramático, en clase.

Es el momento de contrastar alguno de los principios sobre el juego dramático plasmados en el marco teórico dedicado a este asunto, con la percepción que el profesor de lenguas tiene al respecto.

Pregunta 3. Da tu opinión sobre las siguientes afirmaciones

Equivalencias: Totalmente en desacuerdo: A En desacuerdo: B

Ni de acuerdo ni en desacuerdo: C De acuerdo: D Totalmente de acuerdo: E

\begin{tabular}{|l|c|c|c|c|c|}
\hline \multicolumn{1}{|c|}{ Cuestionario } & $\begin{array}{c}\mathrm{A} \\
\mathrm{n}(\%)\end{array}$ & $\begin{array}{c}\mathrm{B} \\
\mathrm{n}(\%)\end{array}$ & $\begin{array}{c}\mathrm{C} \\
\mathrm{n}(\%)\end{array}$ & $\begin{array}{c}\mathrm{D} \\
\mathrm{n}(\%)\end{array}$ & $\begin{array}{c}\mathrm{E} \\
\mathrm{n}(\%)\end{array}$ \\
\hline $\begin{array}{l}\text { 1 Para llevar a cabo ejercicios relacio- } \\
\text { nados con el juego dramático, el profe- } \\
\text { sor debe tener conocimientos especiali- } \\
\text { zados en técnicas teatrales. }\end{array}$ & $2(8.7)$ & $11(47,8)$ & $5(21,7)$ & $4(17,4)$ & $1(4,4)$ \\
\hline $\begin{array}{l}\text { 2 Las actividades relacionadas con el } \\
\text { juego dramático ayudan a que los estu- } \\
\text { diantes pierdan el pudor. }\end{array}$ & $1(4,4)$ & $2(8,70)$ & $4(17,4)$ & $9(39,1)$ & $7(30,4)$ \\
\hline $\begin{array}{l}\text { 3 Es bueno que el estudiante practique } \\
\text { diferentes roles en el aula, que partici- } \\
\text { pe en situaciones variadas, imitando la } \\
\text { realidad exterior. }\end{array}$ & - & - & - & $12(52,2)$ & $11(47,8)$ \\
\hline
\end{tabular}




\begin{tabular}{|l|r|r|r|r|r|}
\hline $\begin{array}{l}\text { 4 El estudiante se sentirá avergonzado } \\
\text { si se le obliga a realizar actividades tea- } \\
\text { trales. }\end{array}$ & $1(4,4)$ & $1(4,4)$ & $7(30,4)$ & $12(52,1)$ & $2(8,7)$ \\
\hline $\begin{array}{l}5 \text { El estudiante puede percibir el uso } \\
\text { del juego dramático en clase como una } \\
\text { falta de seriedad y de rigor académico. }\end{array}$ & $2(8,7)$ & $1(4,4)$ & $1(4.4)$ & $17(73,8)$ & $2(8,7)$ \\
\hline $\begin{array}{l}\text { 6 El juego en general, y por tanto } \\
\text { el juego dramático, es aplicable en } \\
\text { cualquier actividad y en cualquier } \\
\text { ámbito de la clase de lengua. }\end{array}$ & - & $4(17,4)$ & $8(34,8)$ & $5(21,7)$ & $6(26,1)$ \\
\hline
\end{tabular}

Vistos los resultados, el profesor parece no percibir la necesidad de tener conocimientos especializados, lo que coincide con el punto de vista de nuestro trabajo.

También coincidimos con los participantes del estudio en que el juego dramático es un buen elemento para que los estudiantes rebajen la tensión y pierdan el sentido del ridículo. Pensamos que eso es fundamental en el caso de la corrección fonética, donde el estudiante suele mostrar un enorme pudor a la hora de trabajar con un sistema fónico que no domina.

Los participantes tienen claro que el estudiante debe participar en actividades que tengan que ver con la realidad fuera del aula.

La cuestión 4 está relacionada directamente con la 2 y ahonda en los prejuicios y en la predisposición de los estudiantes a participar en una dinámica de este tipo; el profesor cuenta con la posibilidad de que el estudiante se sienta ridículo con este tipo de actividades en el caso de que forzara su participación en una actividad de juego dramático.

Nuestra experiencia nos indica que el estudiante suele reaccionar bien ante estas propuestas; en el caso de que alguien no se sintiera a gusto, no habría que forzar la situación: a veces viene bien tener un observador.

El uso de las actividades dramáticas, del juego en general, no tiene por qué quitarle seriedad y rigor académico a los ejercicios, pero el profesor tiene en mente la posibilidad de que el estudiante perciba todo lo contrario.

Parece que los entrevistados no tienen claro hasta qué punto estas actividades se pueden aplicar en todos los contenidos del aula de lengua, concretamente en el aula de ELE.

\section{Conclusiones}

Se puede concluir que los profesores entrevistados están abiertos a la utilización del juego dramático en el aula, que lo consideran eficaz y motivador, pero la mayoría ven mucho más fácil la aplicación del estilo convencional de enseñanza, basado en la utilización de manuales y ejercicios muy dirigidos. Asimismo, se da la circunstancia de que la mayoría de los profesores no utiliza, de una forma consciente al menos, el juego dramático en clase.

De todos modos, se percibe el carácter ecléctico del profesor de lenguas en sus comentarios sobre la oportunidad de combinar ambos estilos; por ello, condiciona el uso del 
juego dramático al aula y sus características, así como a la edad, la cultura y la personalidad del estudiante.

Por último, a los profesores les queda claro que no es necesario una preparación teórica especializada en técnicas teatrales para poder llevar a cabo ejercicios relacionados con el juego dramático. En cuanto a la posibilidad de llevarlas al aula, los participantes en este estudio reconocen su valía, pero, en general, plantean límites a su uso.

\section{Bibliografía}

Alonso, J. (1997). Motivar para el aprendizaje: teoría y estrategias. Barcelona: Editorial Edebé.

Alonso, M. y Prieto, R. (2011). Embarque B1. Madrid: Edelsa.

Álvarez, M. (Directora) (2000). Sueña 3. Madrid: Anaya

Arroyo, C. (2003). La dramatización y la enseñanza del español como segunda lengua. Madrid: Comunidad de Madrid.

Bercebal, F. (1998). Un taller de drama. Ciudad Real: Ñaque Editora.

Berry, C. (1973). La voz y el actor. Barcelona: Alba Editorial.

Boquete, G. (2012). El uso del juego dramático en el aula de lenguas: las destrezas orales. Tesis doctoral. Universidad de Alcalá.

Brown, H. D. (1994). Teaching English by Principles: An Interactive Approach to Language Pedagogy. New York: Prentice Hall.

Castrillón, J.M. (1998). "Para un desarrollo integral de la capacidad comunicativa, juego dramático y exposición oral", en Textos de didáctica de la Lengua y de la Literatura, $n^{\circ} 18$. FPCEE.

Castro, F., Díaz, P. y Rodero, I. (2005). Español en marcha A2. Madrid: SGEL.

Cervera, J. (1996). La dramatización en la escuela. Madrid: Bruño.

Cómitre, I. y Valverde, J.M. (1996). "Desarrollo de la competencia oral de la L2 a través de actividades dramáticas", en Propuestas metodológicas para la enseñanza de las lenguas extranjeras. Texto dramático y representación teatral. Universidad de Granada.

Del Hoyo, M., Dorrego, L. y Ortega, M. (2006). Propuestas para dinamizar la clase de ELE. Madrid: Edelsa.

Dorrego, L. (1997). Técnicas dramáticas para la enseñanza del español. Universidad de Alcalá.

Eines, J. (1980). Teoría del juego dramático. Madrid: Servicio de Publicaciones del Ministerio de Educación.

Eines, J. y Mantovani, A. (1997). Didáctica de la dramatización. Barcelona: Gedisa.

Fernández, S. (1997). "Aprender como juego. Juegos para aprender español”, en Las actividades lúdicas en la enseñanza de ELE, Revista Carabela $n^{\circ} 41$. Madrid: SGEL.

Fons, M. (2010). "Leer y escribir", en Leer para aprender. Madrid: Ministerio de Educación.

Forment, M. (1997). "La verbalización de la gestualidad en el aprendizaje de ELE”, en Frecuencia ELE, $n^{\circ}$ 4. Madrid: Edinumen.

Hayes, S.K. (1984). Drama as a Second Language: A practical guide for language teachers. Cambridge: National Extension College.

Herans, C. (1983). Teatro, imagen y animación. Barcelona: Laja.

Laferrière, G. (2001). Prácticas creativas para una enseñanza dinámica. La dramatización como herramienta didáctica y pedagógica. Ciudad Real: Editorial Ñaque. 
Laferrière, G. y Motos, T. (2003). Palabras para la acción. Términos de teatro en la educación y la intervención sociocultural. Ciudad Real: Ñaque.

Magariño, C. (1996). "Técnicas dramáticas y su aplicación a la enseñanza de una segunda lengua: La caracterización y la improvisación”, en Propuestas metodológicas para la enseñanza de las lenguas extranjeras. Texto dramático y representación teatral. Universidad de Granada.

Menéndez, M. (coord.) (2009). Prisma A1. Madrid: Edinumen.

Motos, T. y Tejedo, F. (1987). Prácticas de Dramatización. Barcelona: Humanitas.

Motos, T. (1993). "Las técnicas dramáticas: procedimiento didáctico para la enseñanza de la lengua y la literatura”, en Enseñanza: anuario interuniversitario de didáctica. Años 1992-1993, n. 10-11. Editora de la Universidad de Salamanca.

Motos, T. (2003). "Bases para el taller creativo expresivo", en A. Gervilla, Creatividad Aplicada. Una apuesta de futuro. Madrid: Dykinson.

Navarro, M.R. (2005). El valor pedagógico de la dramatización: su importancia en la formación inicial del Profesorado. Tesis doctoral. Universidad de Sevilla.

Robles, M.M. (2007). Drama y teatro. La representación de una pieza teatral en la clase de E.L.E. Tesis doctoral. Universidad de Salamanca.

Ruiz, R. y Martínez, J.A. (ed.) (1996). Propuestas metodológicas para la enseñanza de lenguas extranjeras. Texto dramático y representación teatral. Granada: Universidad de Granada. 